\title{
Effectiveness of stratospheric solar-radiation management as a function of climate sensitivity
}

\section{Citation}

Ricke, Katharine L., Daniel J. Rowlands, William J. Ingram, David W. Keith, and M. Granger Morgan. 2011. "Effectiveness of Stratospheric Solar-Radiation Management as a Function of Climate Sensitivity." Nature Climate Change 2 (2) (December 18): 92-96. doi:10.1038/ nclimate1328.

\section{Published Version}

doi:10.1038/nclimate1328

\section{Permanent link}

http://nrs.harvard.edu/urn-3:HUL.InstRepos:13406231

\section{Terms of Use}

This article was downloaded from Harvard University's DASH repository, and is made available under the terms and conditions applicable to Open Access Policy Articles, as set forth at http:// nrs.harvard.edu/urn-3:HUL.InstRepos:dash.current.terms-of-use\#OAP

\section{Share Your Story}

The Harvard community has made this article openly available.

Please share how this access benefits you. Submit a story.

\section{Accessibility}




\section{Effectiveness of stratospheric solar radiation management as a}

2 function of climate sensitivity

4 Katharine L. Ricke* ${ }^{1}$, Daniel J. Rowlands ${ }^{2}$, William J. Ingram ${ }^{\mathrm{s} 2,3}$, David W. Keith ${ }^{4}$, and

5 M. Granger Morgan ${ }^{5}$

6

7 1. Department of Global Ecology, Carnegie Institution for Science, 260 Panama Avenue,

8 Stanford, CA 94305, USA.

9 2. Atmospheric, Oceanic and Planetary Physics, University of Oxford, Clarendon

10 Laboratory, Parks Road, Oxford, OX1 3PU, UK.

11 3. Also at Met Office Hadley Centre, FitzRoy Road, Exeter, EX1 3PB, UK.

12 4. John F. Kennedy School of Government, Harvard University, 79 JFK Street,

13 Cambridge, MA 02138, USA.

14 5. Department of Engineering \& Public Policy, Carnegie Mellon University, 5000 Forbes

15 Avenue, 129 Baker Hall, Pittsburgh, PA 15213, USA.

16

17 *kricke@stanford.edu

18 
19 If implementation of proposals to engineer the climate through solar radiation

20 management (SRM) ever occurs, it is likely to be contingent upon climate sensitivity.

21 However, modeling studies examining the effectiveness of solar radiation

22 management (SRM) as a strategy to offset anthropogenic climate change have used

23 only the standard parameterizations of Atmosphere-Ocean General Circulation

24 Models (AOGCMs) that yield climate sensitivities close to the Coupled Model

25 Intercomparison Project (CMIP) mean. Here, we use a perturbed physics ensemble

26 modeling experiment to examine how the response of the climate to SRM

27 implemented in the stratosphere (SRM-S) varies under different greenhouse gas

28 (GHG) climate sensitivities. When SRM-S is used to compensate for rising

29 atmospheric concentrations of GHGs, its effectiveness in stabilizing regional

30 climates diminishes with increasing climate sensitivity. However, the potential of

31 SRM-S to slow down unmitigated climate change, even regionally, increases with

32 climate sensitivity. On average, in variants of the model with higher sensitivity,

33 SRM-S reduces regional rates of temperature change by more than 90 percent and

34 rates of precipitation change by more than 50 percent.

36 The Royal Society has defined solar radiation management (SRM) as techniques that

37 "attempt to offset effects of increased greenhouse gas concentrations by causing the Earth

38 to absorb less solar radiation" [1]. The most plausible large-scale method is to increase

39 the loading of light-scattering aerosols in the stratosphere (SRM-S) [1]. A number of

40 AOGCM modeling studies suggest that SRM can compensate for many of the

41 temperature and precipitation changes associated with global warming, even at the 
42 regional level [2-4], though these regional compensatory effects are not uniform [4,5].

43 These previous studies have used models in which the climate's equilibrium sensitivity to

44 greenhouse gas forcing (henceforth, CS) reflects near-median estimates of CS. However,

45 both observationally-constrained and expert-elicited estimates of CS have a substantial

46 "high tail" [6,7] and it is arguably more likely that if SRM is deployed it will be because

$47 \mathrm{CS}$, and the impacts from climate change, turn out to be higher than current best

48 estimates. Here we examine the effectiveness and side effects of SRM-S across a range

49 of CS to check if use of the mean CS biases our understanding of SRM.

50 Evaluating the effectiveness of SRM-S requires first specifying the conditions in

51 which it might be implemented and the effects that would be desired. There are various

52 scenarios under which SRM might be employed. From a conventional policy viewpoint

53 in which SRM is one of a portfolio of strategies alongside mitigation and adaptation, it

54 could be used to minimize net social costs of climate change [8,9]. Alternatively, SRM is

55 often framed as disaster insurance to be employed in case of the "extreme warming" that

56 would occur under high CS [10] (and which may bring about "catastrophic" changes such

57 as rapid deterioration of the Greenland ice sheet or large releases of methane from

58 thawing permafrost [11]).

59 To investigate how SRM-S might be used to counterbalance future GHG-induced

60 climate change in model variants with high CS that are also consistent with recent

61 observed climate change, we perform a "perturbed physics" ensemble (PPE) modeling

62 experiment with the HadCM3L AOGCM [12-15]. Like other PPEs [16,17], we simulate

63 past and future climate scenarios using a wide range of model parameter combinations

64 that both reproduce past climate within a specified level of accuracy but simulate future 
65 climates with a wide range of climate sensitivities. We chose 43 members ("model

66 variants") from a subset of the 1,550 from the British Broadcasting Corporation (BBC)

67 climateprediction.net (cpdn) project that have data that allow restarts (see Methods,

68 Supplementary Methods and Supplementary Figure S1). [12,13]

69 Anthropogenic emissions were modeled using a mid-range standard emissions

70 scenario, SRES A1B [18]. SRM-S is simulated in the model by specifying a globally

71 uniform aerosol optical depth (AOD). The simulations run through 2000-2080 with

72 SRM-S forcings applied from 2005. A first cpdn experiment using HadCM3L's standard

73 physical parameters (i.e., the "standard physics" model variant) to look at global and

74 regional responses to 135 different potential SRM-S scenarios [3] showed that, even

75 regionally, changes to stratospheric AOD produce approximately colinear temperature

76 and precipitation responses. Using the SRM-S scenarios that best stabilized global

77 temperature in that experiment, we analyze the effects of four SRM-S scenarios (no-,

78 low-, medium-, and high-SRM) to simulate with the PPE. The low-, medium- and high-

79 SRM scenarios are designed to approximately counteract rising radiative forcing from

80 anthropogenic emissions and stabilize global mean temperature within $1^{\circ} \mathrm{C}$ relative to

81 present day in all model variants (see Methods, Supplemental Methods and Figure S2).

82 The no-SRM scenario used a constant stratospheric AOD corresponding to mean natural

83 volcanic activity in the recent past. [19]

84 Figure 1 shows five-year-running-mean global-mean surface air temperature and

85 precipitation rates for each model variant for the no-SRM, low-SRM and high-SRM

86 scenarios. SRM cannot simultaneously compensate for the impacts of rising greenhouse

87 gases on both temperatures and the hydrological cycle. Most of the effect of either SRM

Ricke et al., November $2011 \quad$ Draft, Do Not Cite 
88 or GHGs on mean precipitation is via temperature, but if their effects on temperature are

89 made to cancel, changes in mean precipitation are driven by the direct effects of their

90 radiative forcings, both of which reduce precipitation (by reducing surface radiative

91 heating and reducing tropospheric radiative cooling, respectively) [20, 21]. Under the

92 no-SRM scenario, global-mean temperature and precipitation increased with all model

93 variants. While results vary, both high- and low-SRM yield relatively stable

94 temperatures after 2020 and show decreasing precipitation.

95 To analyze the regional impacts of different levels of SRM-S we examined mean

96 temperature and precipitation anomalies over land in 23 "Giorgi regions" [22] (responses

97 over the ocean are not displayed but tend to be similar). Results are presented for each

98 PPE model variant using the projected warming without SRM-S from 2000 to 2050 as the

99 independent variable. The projected warming is correlated with CS and the results of

100 analyses presented in the following sections are the same if CS is used as the independent

101 variable.

102 As an example of how regional responses to greenhouse gas and SRM-S forcings

103 vary among model variants, Figure 2 shows decadal-mean temperature and precipitation

104 changes between 2000 and 2050, normalized by the ensemble-mean inter-annual

105 variability of control climates unperturbed by greenhouse gases or SRM, for just two

106 regions and two model variants: the standard physics variant $\left(\Delta \mathrm{T}_{2050}=2.1 \mathrm{C}\right)$ and the

107 ensemble's highest-warming variant $\left(\Delta \mathrm{T}_{2050}=4.1 \mathrm{C}\right)$.

108 With both model variants, Region 1 gets warmer and wetter under A1B, while

109 Region 2 gets warmer and drier. When SRM-S is used, both regions move back towards

110 their baseline climate states in both model variants. In the standard physics model variant, 
111 with the right amount of SRM-S, each region could return almost exactly to its 2000

112 baseline for both annual-average temperature and precipitation although the amount of

113 forcing required is different for the two regions. In the high CS model variant, the closest

114 each region can return to its baseline climate state is approximately one standard

115 deviation. (These data points were selected for illustrative purposes, but are reasonably

116 representative. Not all low sensitivity model variants return Region 1 and Region 2 so

117 close to the origin, and some regions cannot be simultaneously returned to their baseline

118 values of temperature and precipitation even in the standard physics model variant. See

119 Supplementary Figures S3 and S4.)

120 The ensemble design allows analysis of the relationship between various regional

121 measures of SRM-S efficacy and the overall global warming or CS of the model variant.

122 Regional SRM-S efficacy-defined here as the fractional extent that SRM-S can return

123 regional climates from the no-SRM case toward the baseline-can be expressed in both

124 relative and absolute terms. These measures are averaged for presentation using three

125 different weightings: each region is unweighted; each is weighted by its population; or

126 each is weighted by its economic output. [23]

127 To assess the diversity of likely regional preferences for the amount of SRM-S,

128 we first consider OD*, the change in optical depth that returns the region's climate closest

129 to its baseline (the origin in Figure 2) in terms of combined interannual standard

130 deviations of temperature and precipitation. We also consider regional anomalies (the

131 variability-normalized regional temperature, precipitation, and combined temperature and

132 precipitation changes) for variously weighted mean-OD* and the ratio of regional

133 anomalies at global-mean-OD* to those associated with no SRM.

Ricke et al., November $2011 \quad$ Draft, Do Not Cite 
134 Analyzing precipitation rather than, for example, soil moisture to evaluate the

135 effect of SRM-S on the hydrological cycle does not seem to result in a systematic

136 overestimation of its efficacy. For example, as the amount of SRM-S increases, regional

137 precipitation anomalies associated with anthropogenic emissions, are generally

138 'overcorrected' (SRM changing the sign of the anomaly compared with the no-SRM

139 case) before runoff (precipitation minus evaporation) anomalies are.

140 Precipitation and temperature changes, albeit very important, are only two of the

141 many variables likely to have climate related impacts. The potential for moderating

142 effects such as sea level rise and ice sheet melt (while more difficult to accurately model

143 in AOGCMs) will also be relevant to decisions by some parties about whether to

144 implement SRM-S. As such, our SRM efficacy metrics are useful indicators of tradeoffs

145 that occur when attempting to stabilize regional GHG-driven climate changes using

146 SRM-S, but are not definitive normative measures of regional impacts or likely

147 preferences. Because our simulations do not include 'threshold' effects such as collapse

148 of the thermohaline overturning or catastrophic release of methane, our metrics also

149 cannot measure the ability of SRM-S to counteract the type of forcing feedbacks that

150 would occur if certain climate tipping points were surpassed [24] before SRM-S

151 implementation.

152 Ten-year mean values of various efficacy measures against model variant

153 temperature response for decades averaged around 2030, 2050 and 2070 are shown in

154 Figure 3 and in Supplementary Figures S5 and S6. As greenhouse gas concentrations rise,

155 more SRM-S is required to compensate (Figure 3). Mean regional preferences for the

156 amount of optical depth modification (i.e., mean-OD*) are fairly insensitive to modelled

Ricke et al., November $2011 \quad$ Draft, Do Not Cite 
157 CS regardless of weighting. This should be expected physically because a model variant

158 more sensitive to one radiative forcing is generally similarly sensitive to the other

159 radiative forcing and SRM-S is used to cancel roughly the same amount of forcing

160 regardless of the modelled CS. Results are similar using median-OD* rather than mean.

161 Trends for seasonal data are similar, though the economic output weighted slopes do

162 change noticeably because economic output is concentrated in the Northern Hemisphere

163 (not shown).

164 The standard deviation of regional preferences for OD* (Supplementary Figure

165 S7) decreases with modelled temperature response. This should also be expected

166 physically as the smaller variation in the strength of SRM-S would have more impact if

167 climate sensitivity were higher.

168 However, the mean and standard deviation of regional anomalies at mean-OD*

169 increase with modelled warming (Supp Figure S5), again regardless of weighting. On

170 average across the ensemble, at OD* these SRM-modified climates are slightly warmer

171 and drier than their baseline climates, as is physically expected [21,22]. The higher

172 regional anomalies are driven by amplified regional drying in high-CS worlds; there is no

173 statistically significant relationship between modelled warming and the magnitude of

174 regional temperature anomalies with SRM-S set at mean-OD*. As a proxy for regional

175 impacts with SRM, the higher mean anomalies imply that SRM-S is less effective overall

176 as a substitute for mitigation in higher sensitivity worlds - precisely when SRM-S seems

177 most likely to be deployed. Higher standard deviations of regional anomalies in higher

178 CS model variants also suggest interregional heterogeneities associated with an SRM-S

179 substitution would be greater in higher sensitivity worlds.

Ricke et al., November $2011 \quad$ Draft, Do Not Cite 
181 anomalies at mean-OD* to anomalies with no SRM-S decrease with modelled CS and

182 decrease over the length of the simulations (Supp Figure S6). By these measures, SRM-S

183 is more effective and equitable at reducing the risk from climate change when CS is high.

184 From some impacts perspectives, rates of regional climate change matter more

185 than absolute anomalies $[25,26]$. On average, without SRM-S, regional rates of warming

186 and precipitation change are more than twice as high in the ensemble's highest sensitivity

187 model variants as in the lowest sensitivity model variants and are similar in magnitude to

188 the regional rates of change simulated by the same variant between 1996-2005. With

189 SRM-S applied, the rates of temperature change are insensitive to the modelled CS

190 (Figure 4a). Rates of precipitation change are marginally (but statistically significantly)

191 higher in higher CS model variants (Figure 4c), but on average, SRM-S reduces regional

192 rates of temperature change by more than $90 \%$ and rates of precipitation change by more

193 than $50 \%$ in the highest $\mathrm{CS}$ model variants (forecast warming greater than $3.5^{\circ} \mathrm{C}$ ). The

194 ability of SRM-S to reduce rates of change in the face of high CS does not depend

195 strongly on the inter-regional weighting scheme, implying that while divisions between

196 Giorgi regions are socioeconomically meaningless, the average responses of the regions

197 are still meaningful. Effectiveness also does not depend on the decade, implying that the

198 effectiveness of SRM-S in reducing change is roughly independent of when it is

199 implemented.

200 Given the regional heterogeneity of SRM-S effectiveness and the fact that it will

201 only moderate, never eliminate regional climate changes, it is unlikely that all regions

202 would find their local outcomes comparably satisfactory, and many regions may find the 
203 result increasingly unsatisfactory over time. Conceivably some regions will prefer their

204 new climates to those of 2000. In addition there are other risks (such as potential for

205 stratospheric ozone depletion $[27,28]$ ) and imperfections (such as a failure to address

206 ocean acidification [29]) associated with SRM-S which may also vary with CS.

207 We have explored how much existing assessments of SRM-S, by using standard

208 GCMs with near-median CS, may ignore important contingencies. As noted above, a

209 major motivation for studying SRM is to evaluate its potential effectiveness as insurance

210 against higher-than-expected sensitivity of climate to radiative forcing due to greenhouse

211 gases. We find that SRM-S is least effective in returning regional climates to their

212 baseline states and minimizing regional rates of precipitation change under precisely such

213 high CS conditions. On the other hand, given the very high regional temperature

214 anomalies associated with rising greenhouse gas concentrations under high CS, this is

215 also where SRM-S is most powerful in reducing change relative to the no SRM-S

216 alternative.

217

218 METHODS

219 Ensemble Design

220 The standard versions of AOGCMs have generally benefited from considerable tuning:

221 the set of values of model parameters has been developed to give physically-based

222 realistic simulations. A PPE deliberately "detunes" the model, setting parameters to any

223 physically plausible value, to explore uncertainty space. Many of the original 1,550

224 climateprediction. net model variants thus provide a poor simulation of recent observed

225 climate change. We aim to use only model variants that provide a credible simulation of

Ricke et al., November $2011 \quad$ Draft, Do Not Cite 
226 the past 50 years while maintaining a large diversity in the response in 2050. A number

227 of the choices we made in the design are for pragmatic reasons rather than being based on

228 a formal sampling algorithm, since we do not seek to interpret the distribution of model

229 variants in the new ensemble in any probabilistic terms. Several factors were considered

230 in selecting model variant runs.

231 First, we held constant the future solar forcing scenario [30], and the future

232 anthropogenic sulphate emissions trajectory. To avoid discontinuities in the solar forcing

233 at the year 2000 we only consider simulations with a solar forcing very close to the

234 chosen scenario in 2000 . Second, we only used model variants with a relatively stable

235 base climate. We eliminated model variants in which the initial-condition ensemble

236 average of the control simulations exhibited a drift greater than $0.5 \mathrm{~K} /$ century fitted over

237 1960-2080. Finally, we selected model variants through a comparison of the modelled

238 and observed spatio-temporal pattern of temperature change over the past 50 years (see

239 Supplementary Methods).

240 Supplementary Figure S1 plots the goodness of fit between models and

241 observations against simulated warming in 2050 with our forty-three-member PPE

242 ensemble. The colour code for those points indicates the model's calculated equilibrium

243 climate sensitivity from corresponding equilibrium slab ocean simulations, which is

244 correlated with transient warming (see Supplementary Methods).

245 To select a subset of the models for inclusion in the new ensemble that ensured a

246 wide range of responses in the future, models were binned by projected warming in 2050

247 into 10 equally spaced bins spanning the range of responses. In each bin, the model

248 variant with the lowest $r^{2}$ was automatically included, along with 4 others sampled

Ricke et al., November $2011 \quad$ Draft, Do Not Cite 
249 probabilistically (see Supplementary Methods), avoiding duplicates. In the two highest

250 response bins there were less than 5 model variants that met the selection criteria, and

251 hence our selection yielded only 43 model variants.

252 A 10-member initial condition ensemble was generated for each model variant.

253 (see Supplementary Methods) For our analysis, the 430-member ensemble was run for

254 each of the 4 SRM-S scenarios, giving a total of 1720 model simulations.

255

256 SRM Forcings

257 SRM-S activities were simulated by specifying globally uniform variations in

258 stratospheric optical depth. This is distributed in the vertical proportional to the mass of

259 air in each stratospheric level in each level above the tropopause, which is diagnosed for

260 each point and timestep using a lapse-rate-based criterion [31].

261 A baseline SRM-S scenario (medium-SRM) was formulated using the results

262 from the standard physics experiment [3] in which 135 SRM-S scenarios were

263 formulated, designed to offset the net forcings associated with long-lived greenhouse

264 gases, tropospheric sulphur aerosols and tropospheric ozone; and spanning the

265 uncertainties associated with these anthropogenic forcings. The two scenarios which best

266 stabilized global surface air temperature in that experiment according to a least-squares

267 fit analysis were averaged. In the no-SRM scenario, stratospheric AOD was set to 0.01

268 (at 0.55 microns, the reference wavelength [31]), a level approximately equal to mean

269 volcanic activity in the recent past [19], over the entire length of the simulations. The

270 high-SRM-S and low-SRM-S scenarios are the same as the baseline SRM-S scenario

271 except for the addition (0.075) or subtraction (0.015) of a constant amount of optical

Ricke et al., November $2011 \quad$ Draft, Do Not Cite 
272 depth at all points in the simulations (see Supplementary Figure S2 and Supplementary

273 Methods).

274

275 Statistical Analysis

276 For each of the 43 model variants we average output over a 10-member initial condition

277 ensemble to improve the signal-to-noise ratio. All best fits shown were fitted using least-

278 squares regression. (See Supplementary Table S1 for all regression coefficients and

279 corresponding $\mathrm{p}$-values.) The latter are calculated using standard assumptions including

280 Gaussian noise, which may be misleading, particularly in the far tails. We therefore do

281 not specify p-values beyond 2 decimal places.

282

283 Regional Population and Economic Weightings

284 Population and economic output data for the year 2005 were obtained from the Nordhaus

285 G-Econ dataset, which contains gross output and population at a $1^{\circ} \times 1^{\circ}$ resolution and

286 mapped onto the 22 "Giorgi regions," plus New Zealand [23].

288 Acknowledgments The authors thank the climateprediction.net participants for their

289 donations of computing power without which the experiment would not have been

290 possible. We thank M.R. Allen for advice in the design of the experiment, M.I. Thurston

291 and N.R. Massey for deployment of the experiment through the cpdn system and J.B.

292 Moreno-Cruz and two anonymous referees for thorough \& valuable comments on the

293 manuscript. KLR acknowledges support from a US National Science Foundation

294 Graduate Research Fellowship. DJR was supported by a NERC PhD studentship with a

Ricke et al., November $2011 \quad$ Draft, Do Not Cite 
295 CASE award from CEH Wallingford. WJR was supported by NERC contract

296 NE/D012287/1 and EU FP6 contract 036946. KLR, DWK and MGM acknowledge the

297 support of the Climate Decision Making Center (SES-0345798) and the Center for

298 Climate and Energy Decision Making (SES-0949710), both funded by the US National

299 Science Foundation.

300

301 Contributions KLR and DR designed the experiment. KLR performed the data analysis.

302 KLR, DR, WJI, DWK and MGM discussed the results and wrote the paper.

303

304 Competing financial interests The authors declare no competing financial interests.

306 1. The Royal Society, Geoengineering the Climate: Science, governance and

307 uncertainty, 82pp., September 2009. Available on line at:

308 royalsociety.org/WorkArea/DownloadAsset.aspx?id=10768.

309 2. Caldeira, K. \& Wood, L. Global and Arctic climate engineering: numerical model

310 studies. Philos Transact A Math Phys Eng Sci 366, 4039-4056 (2008).

311 3. Moreno-Cruz, J. B., Ricke, K. L. \& Keith, D. W. A simple model to account for

312 regional inequalities in the effectiveness of solar radiation management. Climatic

313 Change Doi: $10.1007 / \mathrm{s} 10584-011-0103-\mathrm{z}$ (in the press)

314 4. Ricke, K. L., Morgan, M. G. \&Allen, M. R. Regional climate response to solar

315 radiation management Nature Geoscience 3, 537-541 (2010).

316 5. Jones, A., Haywood, J., Boucher, O., Kravitz, B., \& Robock, A. Geoengineering by

317 stratospheric $\mathrm{SO}_{2}$ injection: Results from the Met Office HadGEM2 climate model 
318 and comparison with the Goddard Institute for Space Studies ModelE. Atmos. Chem.

319 Phys. Discuss. 10, 7421-7434, (2010).

320 6. Roe, G. H. \& Baker, M. B. Why is climate sensitivity so unpredictable? Science 318, $321 \quad 629-632(2007)$.

322 7. Zickfeld, K, Morgan, M.G, Frame, D. J, \& Keith, D. W. Expert judgments about 323 transient climate response to alternative future trajectories of radiative forcing. Proc.

$324 \quad$ Natl. Acad. Sci. 107, 12451-12456 (2010).

325 8. Wigley, T.M.L. A combined mitigation/geoengineering approach to climate

$326 \quad$ stabilization. Science 314, 452-454 (2006).

327 9. Moreno-Cruz, J. B. \& Keith, D. W. Climate policy under uncertainty a case for 328 geoengineering. (submitted to Climatic Change)

329 10. Victor, D.G. Global Warming Gridlock Ch. 6 (Cambridge University Press, 2011)

330 11. Blackstock, J. J. et al., Climate Engineering Responses to Climate Emergencies

331 (Novim, 2009), archived online at: http://arxiv.org/pdf/0907.5140M.

332 12. Frame, D. J. et al. The climateprediction.net BBC climate change experiment: design

333 of the coupled model ensemble. Proc. R. Soc. A 367, 855-870 (2009).

334 13. Rowlands, D. J. et. al. Predictions of 21 st century warming constrained by recent

335 climate observations. (submitted to Nature Geoscience)

336 14. Gordon, C. et al. The simulation of SST, sea ice extents and ocean heat transports in a

337 version of the Hadley Centre coupled model without flux adjustments. Climate

338 Dynamics 16, 147-168 (2000).

339 15. Allen, M.R. Do-it-yourself climate prediction. Nature 401, 642-642 (1999).

340 16. Murphy, J. M. et al. Quantification of modelling uncertainties in a large ensemble of 
341 climate change simulations. Nature, 430 (7001) 768-772 (2004).

342 17. Stainforth, D. A. et al. Uncertainty in predictions of the climate response to rising

343 levels of greenhouse gases. Nature 433 (7024) 403-406 (2005).

344 18. Nakicenovic, N. et al. IPCC Special Report on Emissions Scenarios. pp 570.

345 (Cambridge University Press, 2000)

346 19. Sato, M., Hansen, J.E., McCormick, M.P. \& Pollack, J.B. Stratospheric aerosol

347 optical depth, 1850-1990. J. Geophys. Res. 98, 22987-22994, (1993).

348 20. Allen, M.R. \& Ingram, W.J. Constraints on future changes in climate and the

349 hydrologic cycle. Nature 419, 224-232 (2002).

350 21. Bala, G., Duffy, P.B. \& Taylor, K.E. Impact of geoengineering schemes on the global

351 hydrological cycle. Proc. Natl. Acad. Sci. U.S.A 105, 7664-7669 (2008).

352 22. Giorgi, F. \& Francisco, R. Uncertainties in regional climate change prediction: a

353 regional analysis of ensemble simulations with the HADCM2 coupled AOGCM.

354 Climate Dynamics 16, 169-182 (2000).

355 23. Nordhaus, W. Geography and macroeconomics: new data and new findings. Proc.

356 Natl. Acad. Sci. U.S.A 103, 3510-3517 (2006).

357 24. Lenton, T.M. et al. Tipping elements in the Earth's climate system. Proc. Natl. Acad.

$358 \quad$ Sci. U.S.A 105, 1786-1793 (2008).

359 25. Leemans, R \& Eickhout, B. Another reason for concern: regional and global impacts

360 on ecosystems for different levels of climate change, Global Environmental Change,

$361 \quad 14,219-228(2004)$.

362 26. Visser, M.E. Keeping up with a warming world; assessing the rate of adaptation to

363 climate change. Proc. R. Soc. B 275, 649-659 (2008).

Ricke et al., November $2011 \quad$ Draft, Do Not Cite 
364 27. Tilmes, S., Garcia, R.R., Kinnison, D.E., Gettelman, A. \& Rasch, P.J. Impact of

365 geoengineered aerosols on the troposphere and stratosphere. J. Geophys. Res. 114,

366 D12305 (2009).

367 28. Kirk-Davidoff, D. B., Hintsa, E. J., Anderson, J. G. \& Keith, D. W. The effect of

368 climate change on ozone depletion through changes in stratospheric water vapour.

$369 \quad$ Nature 402, 399-401 (1999).

370 29. Hoegh-Guldberg, O. et al. Coral reefs under rapid climate change and ocean

$371 \quad$ acidification. Science 318, 1737-1742 (2007).

372 30. Solanki, S. K. \& Krivova, N. A. Can solar variability explain global warming since

373 1970? J. Geophys. Res., 108, 1200 (2003).

374 31. Cusack S., A. Slingo, J.M. Edwards, and M. Wild, 1998; The radiative impact of a

375 simple aerosol climatology on the Hadley Centre GCM. QJR Meteor. Soc. 124,

$376 \quad 2517-2526$.

Ricke et al., November $2011 \quad$ Draft, Do Not Cite 
377 Figure 1. Time series of temperature and precipitation of the no-SRM, low-SRM and

378 high-SRM scenarios examined, with initial condition sub-ensembles averaged for each of

379 the 43 PPE model configurations analyzed. (a) Five-year running-mean global mean

380 near-surface (1.5 m) air temperature, and (b) five-year running-mean global mean

381 precipitation rate, all displayed over the length of the 80 model-year simulations.

383 Figure 2. Example of regional responses to A1B and SRM-S forcings in units of standard

384 deviations for two model variants and two regions. Region 1 is Eastern North America;

385 Region 2 is Southern Europe/Northern Africa. Blue-edged points show the no-SRM

386 (black-centre), low-SRM (green-centre) and high-SRM (magenta-centre) responses for

387 the standard physics model variant $\left(\Delta \mathrm{T}_{2050}=2.1 \mathrm{C}\right)$. Orange-edged points corresponding

388 responses for the ensemble's highest sensitivity model variant $\left(\Delta \mathrm{T}_{2050}=4.1 \mathrm{C}\right)$.

389 Temperature and precipitation anomalies are the difference between ten-year averages

390 centered on 2050 and 2000, divided by the interannual variability of the control climate.

391 Arrows indicate the trajectory as SRM-S increases.

393 Figure 3. Mean regional values of OD*, the amount of optical depth modification that

394 returns each regional climate closest to its baseline state (the origin in Figure 2), plotted

395 against 2050 forecast warming of the model variant for decadal means about 2030, 2050

396 and 2070. Points show the mean-OD* for each model variant when equal weight has

397 been given to each of the 23 regions. Solid lines show best fits to these points. Dashed

398 and dotted lines show best fits to points (not shown) that result if each geographic region

399 is weighted by its economic output (dotted) or by its population (dashed).

Ricke et al., November $2011 \quad$ Draft, Do Not Cite 
401 Figure 4. The mean value of the absolute values of regional rate of change (a and c) and 402 standard deviation of regional rates of change (b and d) for temperature (a-b) and 403 precipitation (c-d), shown for both the medium-SRM (see Methods) and no-SRM 404 scenarios for decadal intervals centered on 2030 (red), 2050 (black) and 2070 (blue), 405 plotted against model forecast warming. In the case of precipitation, points and best-fit 406 lines for the No-SRM simulations are shaded more lightly to distinguish them from the 407 medium-SRM simulations. 


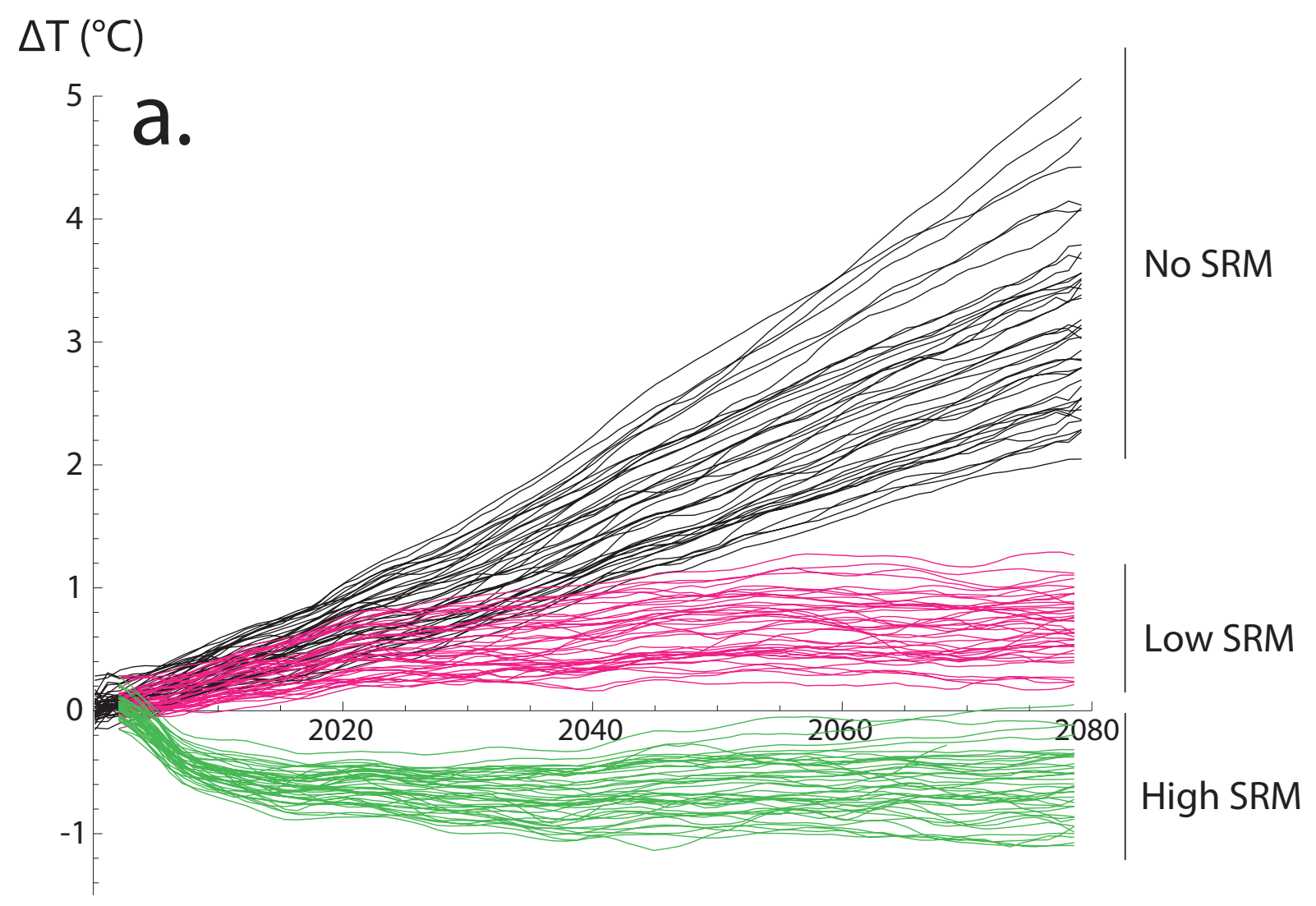

$\Delta \mathrm{P}(\%)$

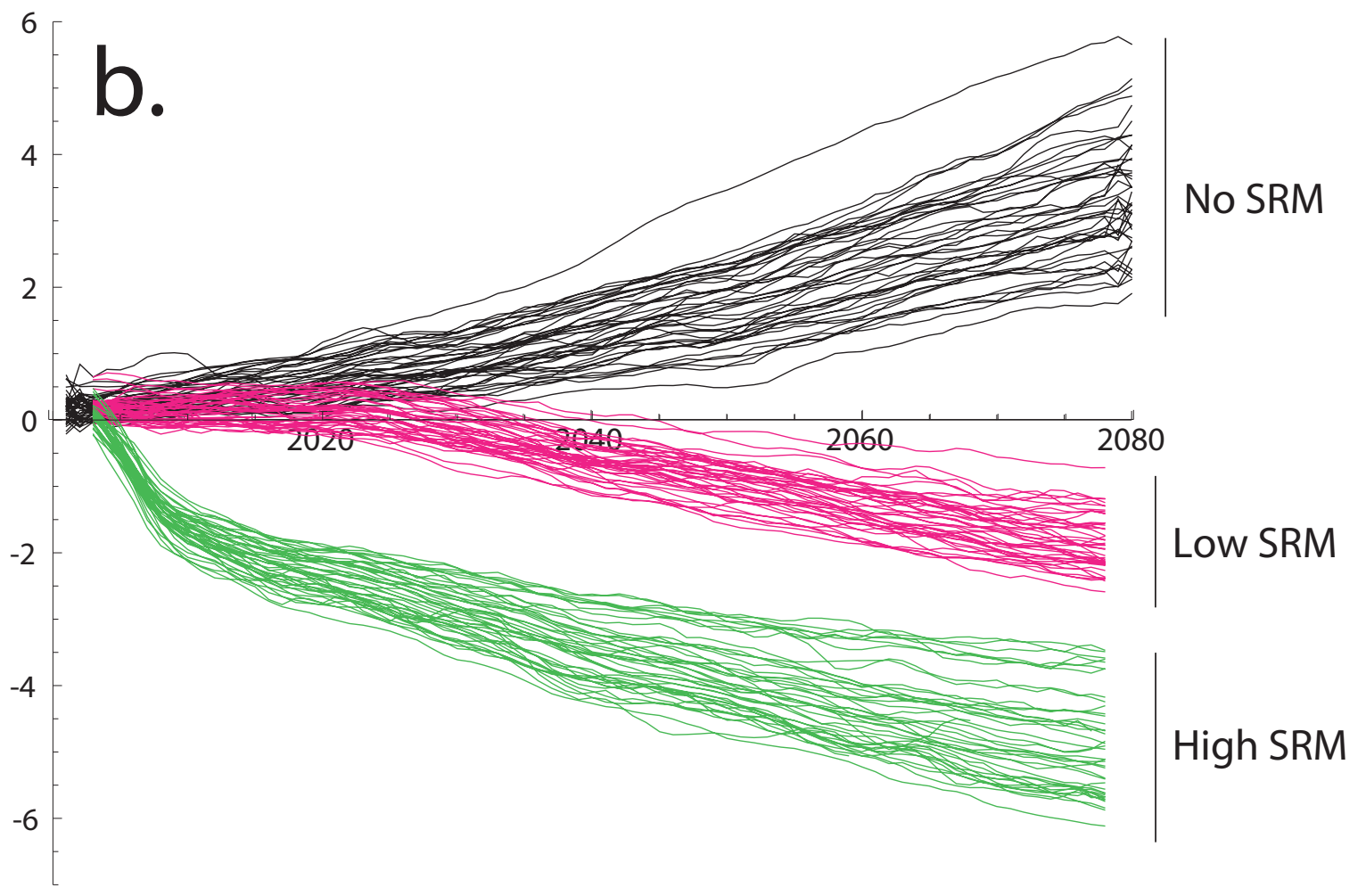


$\Delta$ Precipitation

(standard deviations)

$\nabla$ Region 1

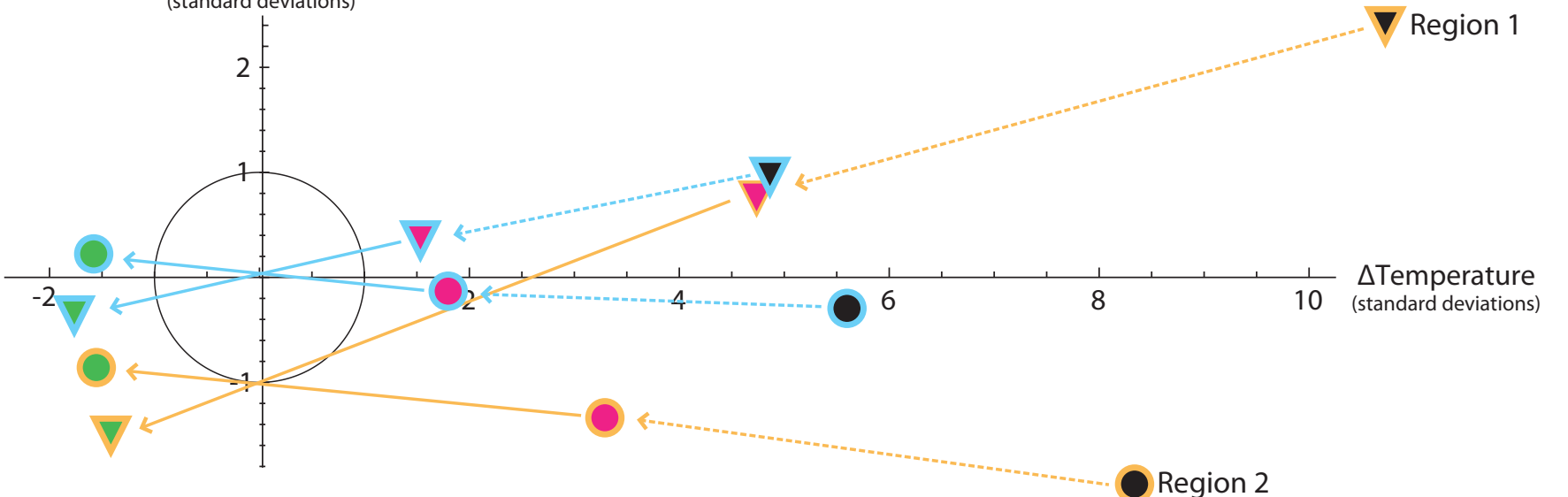

Region 2

Region 1
Region 2

High-SRM

Low-SRM

No-SRM

Standard-Physics

High-Response 


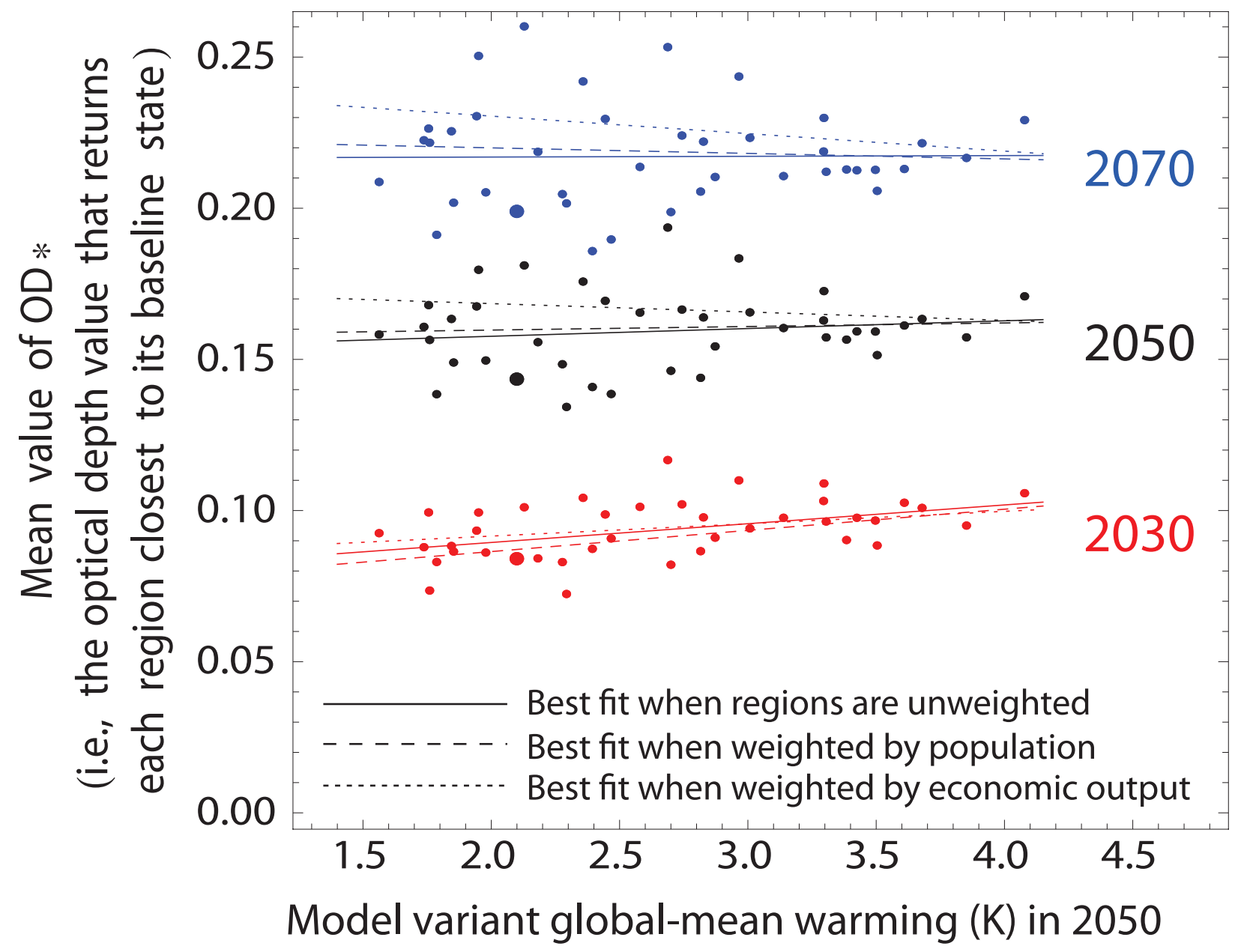



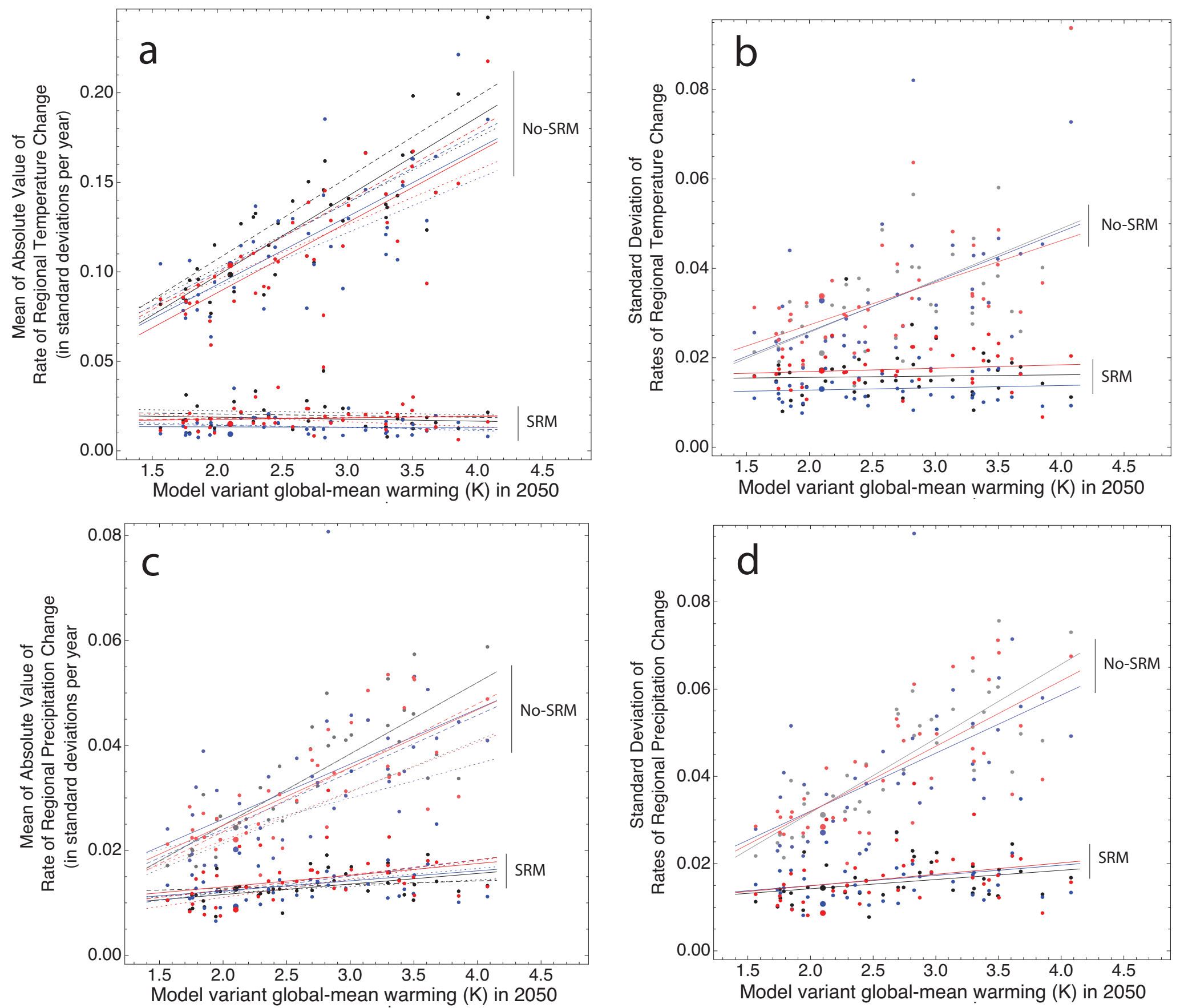